\title{
VISUAL ASSESSMENT AND SHORT TERM NEONATAL OUTCOME OF PRETERM INFANTS
}

S. Huseynova ${ }^{1}$, L. Haataja ${ }^{2}$, S. Hasanov $^{1}$, S. Alasgarova ${ }^{1}$

${ }^{l}$ Neonatology Department, Azerbaijan Medical University, Baku, Azerbaijan, ${ }^{2}$ Department of Pediatric Neurology, University of Turku and Turku University Hospital, Turku, Finland

Background: Visual system is far more complex than optic radiations and primary visual cortex.

Aims: To determine whether impaired visual function of preterm infants at an early neonatal period is associated with neurodevelopmental abnormalities at three months of age.

Methods: 20 term, 40 low birth weight (LBW) and 36 very low birth weight (VLBW) infants born and admitted to NICU at the Azerbaijan Medical University Hospital were recruited to the study. Structured scorable visual assessment was performed to all study infants at an early neonatal period. Cranial ultrasound (US) was performed at the 2-nd and 6-th days of life with 5 and 7,5 $\mathrm{MHz}$ vector transducers. The neurological assessment was performed according to Dubowitz/Ballard scale during neonatal period and at three months of corrected age.

Results: There was no difference between visual function of term and LBW infants. Poorer visual performance was seen in term/LBW infants with hyperbilirubinemia, but there were no other systematical findings between visual function and brain US findings or deviant neurological findings. In contrast, poor visual performance of VLBW infants was accompanied with abnormal neurological findings during both neonatal and postneonatal period at three months of corrected age.

Conclusions: Visual dysfunctions in early neonatal period of VLBW infants is associated with deviant neurological findings in short term follow-up. Structured neonatal visual assessment provides an additional predictive tool for VLBW infants. 